\title{
Are editors of scientific journals doing justice?
}

\author{
Om Prakash Yadava ${ }^{1}$ \\ Published online: 25 January 2020 \\ (C) Indian Association of Cardiovascular-Thoracic Surgeons 2020
}

We deliberated on how scientific is science in our July, 2019 issue of Indian Journal of Thoracic and Cardiovascular Surgery (IJTC) and looked at the pit falls of statistical testing and peer review system [1]. A further corroboration of our take on the issue is provided by a new systematic review of 93 randomised controlled trials (RCTs) with statistically nonsignificant primary outcomes published in six high impact journals, which found that in $57 \%$ of abstracts and $67 \%$ of the main texts, there was a 'spin' from the actual findings to an attempted more favourable outcomes, thus raising doubts on their touted authenticity and rigour [2]. Spin was defined as ".... manipulation of language to potentially mislead readers from the likely truth of the results ....." [2]. The commonly used spin strategies that the authors of the study identified were pivoting to statistically significant secondary results, use of within-group comparisons and creation of special sub-groups to suit the desired outcomes. One may also interpret statistically non-significant primary results as numerically significant and claim their beneficial effect. This often also involves juglary of the language to distract from statistically insignificant primary outcomes to statistically significant secondary results. Another commonly used strategy is to change from 'intention-to-treat' to 'per-protocol' analysis and vice versa - a case in point, the recent Arterial Revascularisation Therapy (ART) Trial [3]. The 'intent-to-treat' analysis, which was the pre-specified criteria for this trial, showed no benefit of multi-arterial grafting; therefore, the authors put forward the 'as-treated' analysis to justify multi-arterial grafting. And there are examples galore-the recently hotly debated EXCEL Trial [4] and the much-quoted PARTNER 3 Trial [5]. In the former, the definition of myocardial infaction (MI) was tweaked midcourse to suit percutaneous coronary

'Be careful about reading health books. You may die of a misprint' Mark Twain

Om Prakash Yadava

op_yadava@yahoo.com

1 National Heart Institute, New Delhi, India interventions (PCI) and in the PARTNER 3 Trial, reported in as high an impact factor journal as New England Journal of Medicine, the triple end points of mortality, cerebro-vascular accident (CVA) and re-hospitalisation at 1 year were not prespecified, yet reported to justify the study. In fact, the choice of including re-hospitalisation in the composite end-point gave a head start to trans-catheter aortic valve replacement (TAVR) to justify its superiority (hazard ratio, $0.54 ; 95 \%$ CI 0.37 to 0.79 ; $P=0.001)$ to surgical aortic valve replacement (SAVR). Similarly, it was pre-specified with the core trial that 2-year results will be presented, yet 1-year results were projected for 2 years and published exhorting the non-inferiority of TAVR as compared with SAVR in low-risk patients [6].

These are classical examples of the 'spin' that authors may provide to their research. Maybe some of this is done subconsciously or with the benign intent of getting the research published, as most scientific journals are reticent in publishing negative results. This may also be due to the pressure of 'publish or perish' and the governmental guidelines demanding publications for promotion, that authors 'spin' and 'doctor' the results to increase the chances of publication. The industry too may have more than a benign interest in the matter. Imagine how much the sponsoring company of the CORE trial were to gain from getting TAVR approved for low-risk cases by publishing projected 2-year results. However, surprisingly, Khan et al. found no association between the spin and conflict of interest disclosures [2]. In fact, the industryfunded research had a lower proportion of spin than nonprofit-funded research - intuitively making one wonder if there is a 'spin' here too!

Not all of this so called 'Spin' may be with fraudulent or malafide intent. Yet, the stark and the gory reality is that it was present and the fact that it may be introduced deliberately, albeit rarely, cannot be discounted. The latter 'spin' provided in the 1960s (purportedly doctored by the soda giants) led to a wild goose chase of fats being the bad boys for atherosclerosis, totally ignoring, and in fact, surreptitiously endorsing, high carbohydrate intake. 
Another moot question that begets an answer is are the peer reviewers and the editor-in-chiefs of the journals doing their job with due diligence? Deputy editor of JAMA Network Open, Dr. Stephan D. Fihn, in an editorial claim that they met twice weekly and 'almost invariably there are one or more manuscripts for which the authors have placed the results in a more favourable light than can be considered justified' [7]. But in how many journals is this practice followed and practical? The editors are practicing surgeons and the editor's job is a secondary pastime. Most editors do not read the manuscripts in details and will not have time to detect the 'spin'. Getting a good peer reviewer, who does the job with sincerity, is becoming increasingly difficult for even the highest impact factor journals. Even debates have started - should peer review be a paid activity and the compensation linked to the diligence with which the peer review is carried out. It is therefore for the professional associations to take a call among the members that should not the editor's job be restricted to fulltime dedicated individuals, not engaging in clinical practice, and should not peer reviewer activity be made a compensated and financially attractive proposition. It is only then, not only the issue of 'spin' fraudulent research and scientific impropriety will be tackled, but also the quality of the papers and the scientific research improve.

This becomes all the more important as a lot of these studies are picked up by electronic media and widely circulated for consumption of the laity. They do not have the wherewithal to go through statistical jargons and quite often pick the gist of the research from abstract and conclusions, without going into the details. Even we, as medical professionals, must learn how to analyse a scientific paper and eruditely read between the lines. In fact it is only when we 'study' important papers, rather than 'read' them, shall we appreciate some of these nuances of 'spin' to scientific facts. I am seized of my own responsibilities as editor-in-chief of IJTC and pari passu exhort my colleague associate editors and the authors to not fight shy of publishing the negative studies. Dr. Fihn puts it succinctly 'Although negative trials typically do not attract as much attention, they are often critically important in countering erroneous inferences from earlier studies or debunking widely held misconceptions' [7].

May be weltschmerz in me, but beware friends of these rhetorical techniques in published literature. All of us need to have our 'spin detectors' turned to the highest fidelity all the time.

\section{References}

1. Yadava OP. Medicine-Turning on its Head! Indian J Thorac Cardiovasc Surg. 2019;35:431-432.

2. Khan MS, Lateef N, Siddiqi TJ, et al. Level and prevalence of Spin in published Cardiovascular Randomised Clinical Trial Reports with statistically nonsignificant primary outcomes, a systematic review. JAMA Netw Open. 2019;2:e192622.

3. Taggart DP, Benedetto U, Gerry S, et al. Bilateral versus single internalthoracic-artery grafts at 10 years. N Engl J Med. 2019;380: 437-446.

4. Stone GW, Kappetein AP, Sabik JF, et al. Five-year outcomes after PCI or CABG for left main coronary disease. N Engl J Med. 2019;381:1820-1830.

5. Mack MJ, Leon MB, Thourani VH, et al. Transcatheter aortic-valve replacement with a balloon-expandable valve in low-risk patients. $\mathrm{N}$ Engl J Med. 2019;380:1695-1705.

6. Popma JJ, Deeb GM, Yakubov SJ, et al. Transcatheter aortic-valve replacement with a self-expanding valve in low-risk patients. N Engl J Med. 2019;380:1706-1715.

7. Fihn SD. Combating misrepresentation of research finding. JAMA Netw Open. 2019;2:e192553.

Publisher's note Springer Nature remains neutral with regard to jurisdictional claims in published maps and institutional affiliations. 